\title{
Optimisation of a double-centrifugation method for preparation of canine platelet- rich plasma
}

\author{
Hyeok-Soo Shin, Heung-Myong Woo and Byung-Jae Kang ${ }^{*}$ (D)
}

\begin{abstract}
Background: Platelet-rich plasma (PRP) has been expected for regenerative medicine because of its growth factors. However, there is considerable variability in the recovery and yield of platelets and the concentration of growth factors in PRP preparations. The aim of this study was to identify optimal relative centrifugal force and spin time for the preparation of PRP from canine blood using a double-centrifugation tube method.

Methods: Whole blood samples were collected in citrate blood collection tubes from 12 healthy beagles. For the first centrifugation step, 10 different run conditions were compared to determine which condition produced optimal recovery of platelets. Once the optimal condition was identified, platelet-containing plasma prepared using that condition was subjected to a second centrifugation to pellet platelets. For the second centrifugation, 12 different run conditions were compared to identify the centrifugal force and spin time to produce maximal pellet recovery and concentration increase. Growth factor levels were estimated by using ELISA to measure plateletderived growth factor-BB (PDGF-BB) concentrations in optimised $\mathrm{CaCl}_{2}$-activated platelet fractions.

Results: The highest platelet recovery rate and yield were obtained by first centrifuging whole blood at $1000 \mathrm{~g}$ for $5 \mathrm{~min}$ and then centrifuging the recovered platelet-enriched plasma at $1500 \mathrm{~g}$ for $15 \mathrm{~min}$. This protocol recovered $80 \%$ of platelets from whole blood and increased platelet concentration six-fold and produced the highest concentration of PDGF-BB in activated fractions.

Conclusions: We have described an optimised double-centrifugation tube method for the preparation of PRP from canine blood. This optimised method does not require particularly expensive equipment or high technical ability and can readily be carried out in a veterinary clinical setting.
\end{abstract}

Keywords: Dog, Double centrifugation, Platelet-derived growth factor-BB, Platelet-rich plasma, Platelet recovery

\section{Background}

Investigations of the morphologic and biologic features of platelets have established their important role in wound healing through the release of various growth factors. These factors, which include platelet-derived growth factor (PDGF), transforming growth factor beta, vascular endothelial growth factor, epidermal growth factor and osteoconductive proteins (fibrin, fibronectin and vitronectin), are contained in platelet $\alpha$ granules and are released when platelets are activated or destroyed [1-3]. The release of these growth factors into damaged tissue

\footnotetext{
* Correspondence: bjkang@kangwon.ac.kr

College of Veterinary Medicine and Institute of Veterinary Science, Kangwon National University, Chuncheon 24341, Republic of Korea
}

facilitates tissue regeneration by stimulating cell proliferation, cell migration, angiogenesis and vascular ingrowth $[4,5]$. Furthermore, platelets also release antimicrobial, anti-inflammatory and analgesic factors [6-8].

The use of platelet-rich plasma (PRP) to deliver growth factors has emerged as a convenient method for promoting wound healing and tissue regeneration. The autologous nature of PRP preparations makes them safer than other allogenic cell-based regenerative therapies and less expensive. As a result, PRP therapy is seeing widespread use in human medicine, with applications in dentistry, orthopaedic surgery, cardiac surgery, ophthalmology and plastic surgery [9-12]. PRP is also increasingly being used in veterinary medicine [13-15], 
prompting the need for development of simple and reproducible methods for preparation of high-quality PRP for use in veterinary patients.

Despite the popularity and advantages of PRP, there are conflicting reports in the literature regarding its efficacy $[9-14,16]$. These differences may in part be attributable to variations in the composition of PRP preparations with respect to platelet yield, concentrations of beneficial growth factors and the presence of other blood components, particularly leukocytes [17-19]. Tube methods employing differential centrifugation to separate and concentrate platelets are most convenient clinical settings because they are low cost and do not require specific technical expertise. Considerable research effort has been focused optimising PRP protocols reproducible recovery, yield and cellular composition. Most of these studies have focused on PRP prepared from human blood [20-22]; relatively fewer studies have addressed optimisation of PRP for use in veterinary medicine [23, 24]. Several commercial products are available for producing PRP from human blood, but they have been found to produce variable results when used to prepare PRP from canine blood [25].

The purpose of this study was to identify optimal centrifugal force and spin times for the preparation of PRP from canine whole blood using a doublecentrifugation tube method. The goal was to identify run conditions that will reproducibly recover large numbers of platelets capable of releasing large amounts of growth factor when activated and that are relatively free of other contaminating blood components. To determine which run conditions were optimal, preparations were assessed for platelet recovery and yield, concentrations of white blood cells (WBC) and red blood cells (RBC) and the amount of PDGF$\mathrm{BB}$ released from activated platelets.

\section{Methods}

\section{Experimental animals}

Twelve, clinically healthy adult male beagles, bred for research purposes (Dreambio, Seoul, Republic of Korea), were included in this study. Dogs were evaluated at the Kangwon National University Veterinary Teaching Hospital by means of physical examination, thoracic and abdominal radiography, complete blood count (CBC), serum biochemistry profile and blood smear microscopic analysis by a veterinarian and were deemed healthy. All dogs in this experiment had normal blood count and morphological features. They ranged from 14.317.8 months of age. Body weights ranged were 9.3$10.1 \mathrm{~kg}$. The dogs were individually housed in stainless steel cages at conventional housing facilities with controlled temperature $\left(21^{\circ} \mathrm{C}\right)$ and humidity of $55 \%$. They received standard care and water and food. All dogs were under the care of a licensed veterinarian.

\section{Blood collection}

Whole blood was collected from the jugular vein of each dog using a 21-gauge multiple-use needle into blood collection tubes containing $1.5 \mathrm{~mL}$ anticoagulant acid citrate dextrose-A solution (BD Vacutainer system, Plymouth, UK). No more than $90 \mathrm{~mL}$ of blood was drawn from each dog per collection, with at least 4 weeks between collections.

Sampled whole blood was divided into 9-mL aliquots that were centrifuged in $15 \mathrm{~mL}$ conical centrifuge tubes. Centrifugation and analysis of samples was initiated within $1 \mathrm{~h}$ of blood collection.

\section{Preparation of platelet concentrates}

A double-spin preparation method was used for all samples. Samples were centrifuged at room temperature in a Hanil MF-550 centrifuge (HanilScience, Kimpo, Korea) and centrifugal brake was not applied. For the first spin, samples were centrifuged at one of 5 different RCFs $(100,200,300,500$ or $1000 \mathrm{~g})$ for 5 and $10 \mathrm{~min}$ at room temperature (RT), for a total of 10 RCF-time comparisons, with four replications for each comparison. Four replications were conducted on different days using blood drawn from different individuals, and $\mathrm{CBC}$ of both whole blood and PRP1 used in each experiment were performed.

After the first centrifugation, the whole plasma fraction above the buffy coat (PRP1) was transferred to an empty tube, its volume was measured, and after mixing this fraction well, a 1-mL aliquot was taken for CBC.

The second centrifugation step was performed with PRP1 prepared using the first-centrifugation RCF-time condition that recovered the highest percentage of platelets. The PRP1 fraction was divided into 1-mL aliquots, which were centrifuged at RT at either 500, 1000, 1500 or $3000 \mathrm{~g}$ for 10,15 or $20 \mathrm{~min}$, for a total of 12 RCFtime comparisons.

The second centrifugation produced a platelet-poor plasma (PPP) supernatant and a platelet-enriched pellet. A volume of $700 \mu \mathrm{L}$ of PPP supernatant was removed, and the pellet was resuspended in $300 \mu \mathrm{L} \mathrm{PPP}$; this concentrated platelet fraction was designated as PRP2.

\section{Platelet activation and PDGF-BB quantification}

There are so many activated PDGF such as EGF, PDGF and TGF- $\beta$. Because PDGF-BB is not included in the plasma, its concentration correlates well with the quantity of activated platelets. Therefore, PDGF-BB was chosen as an index of platelet activation. PDGF-BB levels were assessed in activated PRP1 and PRP2 samples as well as plasma that had been prepared by centrifuging whole blood at $1000 \mathrm{~g}$ for $5 \mathrm{~min}$. To activate samples, they were activated using $20 \mathrm{mM} \mathrm{CaCl}$ and incubated at $37{ }^{\circ} \mathrm{C}$ for $1 \mathrm{~h}$ followed by $4{ }^{\circ} \mathrm{C}$ for $16 \mathrm{~h}$. Samples were 
then centrifuged at $3000 \mathrm{~g}$ for $20 \mathrm{~min}$ at $18{ }^{\circ} \mathrm{C}$, and the supernatant collected for PDGF-BB measurement.

The supernatant for PDGF-BB measurement was diluted 20-fold with diluent buffer in kit and quantified by enzyme-linked immunosorbent assay (ELISA) using antibodies against canine PDGF-BB (Raybiotech, Norcross, GA, USA) according to the manufacturer's instructions. The detection limits ranged from $4 \mathrm{pg} / \mathrm{mL}$ to $1200 \mathrm{pg} / \mathrm{mL}$, the interassay coefficient was $<12 \%$, and the intraassay coefficient was $<10 \%$.

\section{Haematological analysis}

A CBC of whole blood and PRP1 and PRP2 fractions was carried out using an automated laser flow cytometry haematology analyser (ProCyte Dx, IDEXX, ME, USA).

\section{Statistical analysis}

Statistical analysis was performed using Prism 5.00 software (Graphpad Software Inc. San Diego, CA, USA). Results are presented as mean \pm standard deviation. Statistical significance was assessed using the KruskalWallis test followed by the Mann-Whitney post-hoc test (to compare all pairs in group). $P<.05$ was considered significant for all tests.

\section{Results}

The 12 dogs from which blood was collected weighed between 9.3 and $10.1 \mathrm{~kg}$. No significant weight changes and side effects occurred for any dog during the course of the study.

\section{PRP1 after first centrifugation step}

Table 1 shows the results for the 10 different RCF-time run conditions used for the first centrifugation. The

Table 1 Platelet recovery after total blood centrifugation

\begin{tabular}{lllll}
\hline Run & Parameters & Recovery (\%) & $P$ value \\
\cline { 2 - 4 } & RCF $(\times g)$ & $\begin{array}{l}\text { Centrifugation time } \\
\text { (minutes) }\end{array}$ & & \\
\hline 1 & 100 & 5 & $16.33 \pm 10.92$ & $0.0286^{*}$ \\
2 & 100 & 10 & $38.53 \pm 15.46$ & $0.0286^{*}$ \\
3 & 200 & 5 & $31.05 \pm 14.18$ & $0.0286^{*}$ \\
4 & 200 & 10 & $55.51 \pm 12.14$ & $0.0286^{*}$ \\
5 & 300 & 5 & $42.62 \pm 10.22$ & $0.0286^{*}$ \\
6 & 300 & 10 & $73.00 \pm 12.10$ & 0.1143 \\
7 & 500 & 5 & $72.69 \pm 6.90$ & $0.0286^{*}$ \\
8 & 500 & 10 & $76.62 \pm 8.57$ & 0.1143 \\
9 & 1000 & 5 & $92.28 \pm 4.88$ & - \\
10 & 1000 & 10 & $82.77 \pm 8.35$ & 0.3429 \\
\hline
\end{tabular}

Values expressed as mean \pm standard deviation; $\mathrm{RCF}$, relative centrifugal force. Asterisk $\left.{ }^{*}\right)$ indicates a recovery significantly different $(P<.05)$ from condition 9, which produced the highest recovery percent of platelets recovered from whole blood progressively increased as the RCF increased when centrifugation time was held constant. Platelet recovery also increased when RCF was held constant but centrifugation time increased 5 min to 10 min for every RCF except the $1000 \mathrm{~g}$ condition. The greatest recovery was achieved with run condition 9 (1000 g, $5 \mathrm{~min}$ ). Comparison of mean percent recoveries of run conditions $1-5$ and 7 were all significantly lower than the recovery of condition 9 . The mean percent platelet recoveries for conditions $6,8,9$ and 10 were not significantly different. Furthermore, only $8.18 \pm 0.98 \%$ of the whole blood WBCs remained in PRP1 obtained through condition 9 , which was not significantly different from the WBC recovery rate of PRP1 obtained under conditions $1-8$, and it was significantly lower than that of condition 10. Therefore, because the centrifugation time was shorter, condition 9 was chosen as optimal for the first centrifugation step. This condition was used to prepare PRP1 samples for use in optimising the second centrifugation step. (See Additional file 1)

\section{PRP2 after second centrifugation step}

The purpose of second centrifugation step was to further concentrate platelets by reducing the volume of PPP. To identify the optimal RCF-time run conditions for the second centrifugation, PRP1 prepared by condition 9 (1000 g, $5 \mathrm{~min}$ ) was used. The amount of PRP1 obtained by the first centrifugation varied depending on the packed cell volume of each individual, and mean volume of PRP1 was $4.2 \pm 0.32 \mathrm{~mL}$. The PRP1 fraction was divided into $1-\mathrm{mL}$ aliquots and samples were subjected to 12 different centrifugation conditions to obtain PRP2 with four replications for each comparison.

Table 2 shows the percent recovery of platelets relative to PRP1 and yield (fold increase) relative to whole blood at each run condition. Lowest RCF resulted in less percent recovery. Recovery increased with increasing RCF and duration of centrifugation. Recovery was substantially reduced following centrifugation at $3000 \mathrm{~g}$ for $20 \mathrm{~min}$ due to platelet aggregation. Condition 8 (1500 g, $15 \mathrm{~min}$ ) resulted in both highest recovery and increase in platelet concentration. Statistical analysis revealed that the recovery and yield for condition 8 was not significantly different from results obtained using condition 5 (1000 g, $15 \mathrm{~min})$, condition 9 (1500 g, $20 \mathrm{~min}$ ) or condition 10 (3000 g, $10 \mathrm{~min}$ ). Based on these results, conditions 5, 8, 9 and 10 were chosen for additional assessment and each of these 4 run conditions were replicated 8 times.

Mean percent platelet recoveries relative to PRP1 and yields relative to whole blood under run conditions 5, 8, 9 and 10 are shown in Fig. 1a and b, respectively. Platelet recovery and yield were once again the highest when samples were centrifuged at $1500 \mathrm{~g}, 15 \mathrm{~min}$ (condition 8). The 
Table 2 Platelet yield and recovery after the second centrifugation step

\begin{tabular}{lllllll}
\hline \multirow{2}{*}{ Run } & Parameters & & $P$ value & Recovery (\%) & $P$ value \\
\cline { 2 - 5 } & RCF $(\times g)$ & $\begin{array}{l}\text { Centrifugation } \\
\text { time (min) }\end{array}$ & Yield (fold) & & & \\
\hline 1 & 500 & 10 & $4.42 \pm 0.84$ & $0.0286^{*}$ & $63.21 \pm 4.66$ & $0.0286^{*}$ \\
2 & 500 & 15 & $4.65 \pm 0.76$ & $0.0286^{*}$ & $66.65 \pm 3.64$ & $0.0286^{*}$ \\
3 & 500 & 20 & $5.60 \pm 0.49$ & 0.1143 & $74.50 \pm 1.51$ & $0.0286^{*}$ \\
4 & 1000 & 10 & $5.41 \pm 0.37$ & $0.0286^{*}$ & $72.06 \pm 2.68$ & $0.0286^{*}$ \\
5 & 1000 & 15 & $5.94 \pm 0.36$ & 0.3429 & $82.23 \pm 6.51$ & 0.4897 \\
6 & 1000 & 20 & $5.65 \pm 0.13$ & $0.0286^{*}$ & $78.08 \pm 2.85$ & $0.0286^{*}$ \\
7 & 1500 & 10 & $5.80 \pm 0.48$ & 0.1143 & $81.96 \pm 2.58$ & $0.0286^{*}$ \\
8 & 1500 & 15 & $6.48 \pm 0.46$ & - & $91.70 \pm 4.49$ & - \\
9 & 1500 & 20 & $5.93 \pm 0.28$ & 0.3429 & $86.73 \pm 1.54$ & 0.4897 \\
10 & 3000 & 10 & $5.82 \pm 0.39$ & 0.3429 & $85.06 \pm 3.28$ & 0.1143 \\
11 & 3000 & 15 & $5.75 \pm 0.49$ & 0.3429 & $85.87 \pm 1.65$ & $0.0286^{*}$ \\
12 & 3000 & 20 & $4.34 \pm 1.06$ & 0.1143 & $64.79 \pm 13.73$ & $0.0286^{*}$ \\
\hline
\end{tabular}

Values expressed as mean \pm standard deviation; RCF, relative centrifugal force. Asterisk (*) indicates a recovery or yield significantly different

$(P<.05)$ from condition 8 , which produced the highest recovery and yield

platelet recovery rate for condition 8 was significantly higher than those for conditions 5 and 10 but not higher than that for condition 9. The platelet yield for condition 8 was significantly greater than that for condition 10 , but it was not significantly greater than the yield for conditions 5 and 9 (Fig. 1b). There was no statistically significant difference between conditions 8 and 9, but condition 8 could shorten centrifugation time by $5 \mathrm{~min}$. Based on these results, condition 8 ( $1500 \mathrm{~g}, 15 \mathrm{~min})$ was chosen as optimal for the second centrifugation step.

Table 3 shows the concentrations of platelets, RBC, total WBC and lymphocytes in whole blood and in PRP2 prepared using the centrifugation conditions identified as optimal for platelet recovery and yield (i.e. initial centrifugation of whole blood at $1000 \mathrm{~g}$ for $5 \mathrm{~min}$ to obtain PRP1, followed by centrifugation of PRP1 at $1500 \mathrm{~g}$ for $15 \mathrm{~min})$. This centrifugation protocol recovered approximately $80 \%$ of the platelets from whole blood and increased platelet concentration approximately 6 -fold. The concentration of platelets in the PRP preparation was $1748 \pm 193 \times 10^{3}$ cells $/ \mu \mathrm{L}$, which was $6.1 \pm 0.4$ times higher than the concentration in the original whole blood sample. The PRP preparations contained negligible concentrations of RBCs, and WBC contents were $8.5 \pm 1 \%$ neutrophils, $15.5 \pm 4 \%$ monocytes and $73 \pm 7 \%$ lymphocyte. (See Additional file 1)

\section{PDGF-BB quantification}

To determine the effects of the centrifugation protocol on the level of platelet-associated growth factors in activated fractions, concentrations of a representative

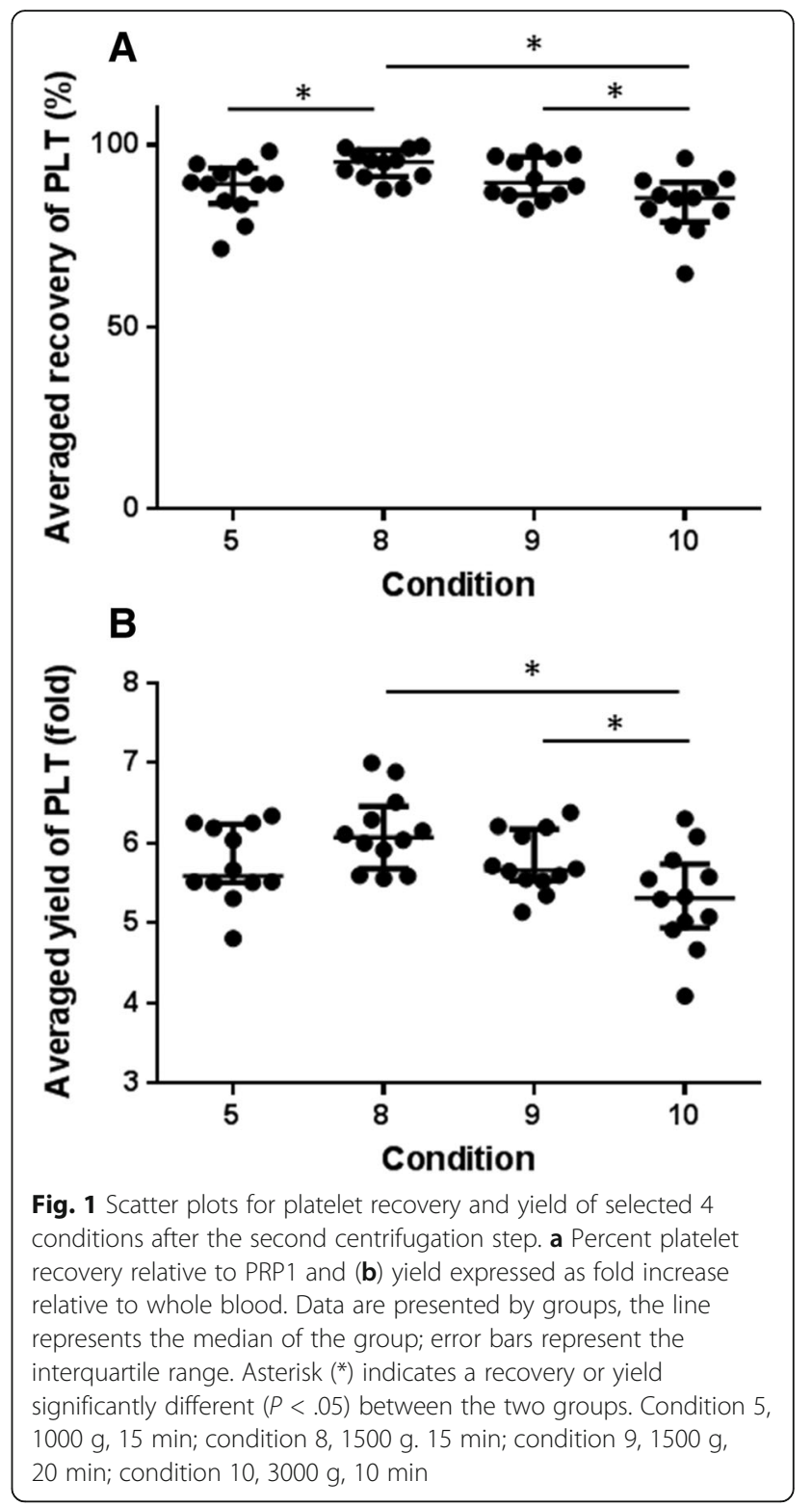

growth factor, PDGF-BB, were measured in plasma, PRP1, and in the various PRP2 fractions prepared using conditions 5, 8, 9, 10 and 11 . Figure 2 shows that condition 8 (1500 g, $15 \mathrm{~min}$ ), which produced the highest recovery and yield of platelets, correspondingly also had the highest concentration of PDGF-BB. PRP2 prepared under condition 8 was the only PRP2 fraction that had a significantly higher PDGF-BB concentration than plasma or PRP1.

\section{Discussion}

In this study, we prepared PRP from canine blood using a double-centrifuge tube method. We found that the following protocol produced the greatest recovery and yield 
Table 3 Descriptive results after double centrifugation

\begin{tabular}{llll}
\hline Variables & Whole blood & PRP1 & PRP2 \\
\hline Red blood cells (cells $\left.\times 10^{6} / \mu \mathrm{L}\right)$ & $5.8 \pm 0.23$ & $0.03 \pm 0.05^{*}$ & $0.065 \pm 0.02^{\#}$ \\
Lymphocytes (cells $\left.\times 10^{3} / \mu \mathrm{L}\right)$ & $2.3 \pm 0.54$ & $1.05 \pm 0.77$ & $3.71 \pm 2.4$ \\
Neutrophil (cells $\left.\times 10^{3} / \mu \mathrm{L}\right)$ & $7.11 \pm 1.67$ & $0.15 \pm 0.17^{*}$ & $0.44 \pm 0.39^{\#}$ \\
Monocyte (cells $\left.\times 10^{3} / \mu \mathrm{L}\right)$ & $0.7 \pm 0.15$ & $0.2 \pm 0.13$ & $0.79 \pm 0.57$ \\
White blood cells $\left(\right.$ cells $\left.\times 10^{3} / \mu \mathrm{L}\right)$ & $10.32 \pm 2.4$ & $1.45 \pm 0.87^{*}$ & $5.09 \pm 3.39^{\#}$ \\
Platelets (cells $\left.\times 10^{6} / \mu \mathrm{L}\right)$ & $289 \pm 35$ & $523 \pm 56^{*}$ & $1748 \pm 193^{\#}$ \\
\hline
\end{tabular}

Values expressed as mean \pm standard deviation $(n=12)$. Asterisk (*) indicates a significant difference $(P<.05)$ between platelet-rich plasma-1 and whole blood. \# indicates a significant difference $(P<.05)$ between platelet-rich plasma-2 and whole blood

of platelets and highest concentrations of PDFG-BB in PRP preparations:

1. Collect blood samples in $10-\mathrm{mL}$ tubes containing $3.2 \%$ sodium citrate to prevent coagulation. Retain a 0.5-mL small aliquot for initial CBC.

2. Centrifuge the blood sample at $1000 \mathrm{~g}$ for $5 \mathrm{~min}$ at RT.

3. Transfer the upper plasma and platelet fraction (PRP1) into a sterile tube and measure the volume. Retain an aliquot for determination of PRP1 platelet concentration.

4. Centrifuge the PRP1 fraction at $1500 \mathrm{~g}$ for $15 \mathrm{~min}$ at RT.

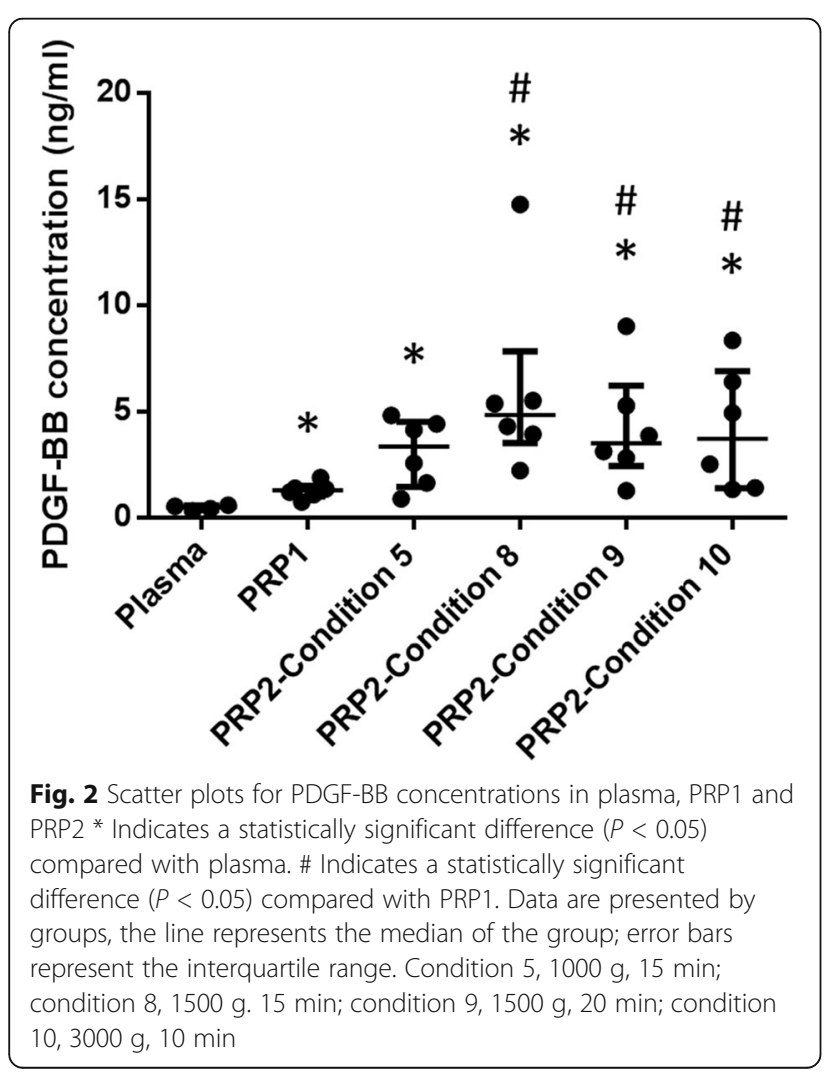

5. Draw off two-thirds of the PPP supernatant and resuspend the platelet-containing pellet PRP2 in the remaining PPP. Measure the PRP2 volume and take an aliquot for determination of PRP2 platelet concentration

The purpose of the first spin is to separate as many platelets as possible from the whole blood. Centrifugation separates blood components on the basis of their density gradients. Platelets are the lightest components, followed by WBCs, and RBCs are heaviest [26]. Therefore, RCF and time must be appropriate to obtain optimal separation of blood components. In this study, a low RCF and short duration of centrifugation was insufficient to obtain good separation of platelets from other blood cells, resulting in low recovery. The highest platelet recovery in conditions 9 and 10, which were the highest RCF conditions, indicated that the 1000-g condition was the most suitable condition to sink RBCs and WBCs and separated platelets at the upper layer. However, in condition 10 , the $\mathrm{WBC}$ recovery rate was also high because the high RCF and long centrifugation time caused the platelet to overlap with the WBC layer.

The purpose of the second spin is to separate platelets from plasma, which is accomplished by pelleting the platelets and discarding most of the PPP supernatant. To maximise platelet recovery and yield, a higher RCF and longer duration of centrifugation than used in the first spin were needed. However, we found that if the RCF time was too high, the recovery and yield were reduced due to platelet aggregation. Aggregated platelets were not resuspended in PPP, thus resulting in substantial platelet count reduction. The blood analyser detects aggregated platelets in PRP2; although the actual numbers of platelets were not decreased, as aggregated (activated) platelets release growth factors during the preparation process, the amount of growth factors in the final product was found to decrease. We concluded that the optimal combination was $1000 \mathrm{~g}$ for $5 \mathrm{~min}$ for the first spin and $1500 \mathrm{~g}$ for $15 \mathrm{~min}$ for the second spin.

The RCFs we found to be optimal for preparation of PRP from canine blood were higher than those found for human blood, although the centrifugation times were similar $[20,21]$. This may be related to haematologic difference between human and dog blood. Canine mean corpuscular volume and mean corpuscular haemoglobin values are lower than human values [27, 28]. These means dog RBCs are smaller and less dense than human $\mathrm{RBCs}$, so relatively higher centrifugal force is required to separate canine blood components.

The optimised protocol increased platelet concentration was 6 - to 7 -fold relative to the initial concentration in whole blood (Table 2), and final platelet concentration was $1748 \pm 193 \times 10^{3}$ platelets $\mu \mathrm{L}^{-1}$ (Table 3 ). The 
American Red Cross defines PRP as $5.5 \times 10^{10}$ platelets/ $50 \mathrm{~mL}\left(1100 \times 10^{3}\right.$ platelets $\left.\mu \mathrm{L}^{-1}\right)$ [29]. Furthermore, accumulated data suggests that the PRP products must have at least $300 \times 10^{3}$ platelets $\mu \mathrm{L}^{-1}$ to be therapeutically effective, which is 4- to 5-fold higher concentration than whole blood [30-34]. These recommendations may not be appropriate to every situation; however, because this protocol produces a high yield of platelets, the concentration can adjusted as necessary by manipulating the ratio of PPP to PRP. Franklin et al. [25] recently conducted a study in which they used five commercially available kits to prepare PRP from canine whole blood. The yield and compositions of the canine PRP prepared using these kits varied considerably. The concentration of platelets in the canine PRP prepared using our optimised protocol was higher than the concentrations achieved with any of the commercial systems. Frye et al. [35] also showed PRP, with platelet and leukocyte concentrations similar to ours through the double-spin method using commercial kits, but its disadvantage was that it was expensive.

Using differential centrifugation to prepare platelet concentrates completely free of other blood cell types is impossible because the specific gravities of the various components slightly overlap [36]. However, our optimised protocol resulted in PRP with negligible number of RBCs compared with other studies [20,21], and only $8.18 \pm 0.98 \%$ of total blood WBC counts were contained in PRP1. Also, the WBC concentration in the final product was 2 -fold lower than the normal range. In particular, the recovery rate of lymphocytes and monocytes from PRP1 obtained with the first spin was higher than that of neutrophils because the lymphocyte and monocyte weights were relatively light and were located in the upper layer near the platelets after centrifugation.

The role of WBC in PRP therapy remains a matter of disagreement in the literature. Some studies have suggested that increased WBC concentrations in PRP can impede tissue regeneration and healing. The presence of WBC in PRP has been associated with increased scar tissue and collagen degradation ex vivo. High WBC concentration may affect negatively with matrix production in tendons [37-39]. To prevent potential negative effects, it has been suggested that platelet preparations only be transfused if they contain fewer than $5 \times 10^{5}$ $\mathrm{WBC} / \mu \mathrm{L}[40]$. However, some research has suggested that some WBC contamination may be beneficial because WBC can increase growth factor secretion. PRP that contained enough number of WBCs was recommended as another therapeutic purpose because WBC triggered growth factor secretion by the tissue being treated with the PRP $[41,42]$. There is not enough data in the literature to determine whether WBCs are beneficial to PRP therapy [29]. Additional studies will be necessary to optimise PRP with regard to their WBC content. Previously, Perazzi et al. [23] performed PRP preparation study using the double-centrifugation method, which is similar to ours. In their study, platelet concentration at least four times the baseline was obtained in 10 out of 12 dogs ( $83.3 \%$ of cases), but WBC concentration was also higher than that at the baseline. Furthermore, they did not measure growth factor concentration in PRP from their protocol; thus, it remained unknown that high platelet number represented high growth factor concentration in PRP.

Among the numerous growth factors present in platelet $\alpha$ granules, PDGF-BB was selected as an index of activated platelets in this study because it is only secreted by activated platelets and not contained in plasma. Thus, its concentration represented the extent to which platelets were activated. PDGF-BB concentration was highest in PRP prepared by the optimised method. Although the PRP that had the highest platelet concentration also had the highest PDGF-BB level, platelet concentration is not the only determinant of growth factor concentration. The type of anticoagulant used during blood collection, the ratio of blood to anticoagulant, the type of activating agent and the activation protocol can all have an influence of the concentration of growth factor in PRP [20]. A previous study showed that platelets released the highest PDGF-BB when they were activated with $\mathrm{CaCl}_{2}$ [43]. Further, the amount of TGF- $\beta$ released by platelets activated by thrombin or $\mathrm{CaCl}_{2}$ was similar [43]. In addition, the process of obtaining autologous thrombin is complicated, but activation using $\mathrm{CaCl}_{2}$ is inexpensive and easy. Therefore, we used $\mathrm{CaCl}_{2}$ to activate platelets. However, we did not measure whether platelets were fully activated in the present study. Among the PRPs with the same number of platelets, the amount of growth factors will depend on the number of activated platelets. To activate as much platelets as possible, various agonist (collagen, ADP and thrombin, etc.) and ratio between PRP and agonist should be further studied.

One potential limitation of using centrifugation to prepare PRP is that there is a risk for bacterial contamination during transfers between tubes. However, this risk can be greatly reduced if transfers are conducted in a laminar flow hood under sterile conditions. Despite this potential drawback, the protocol we have described in this study does not require particularly expensive equipment or high technical ability and can readily be carried out in a veterinary clinical setting.

\section{Conclusions}

The PRP therapy, research and associated-industry are burgeoning fields in veterinary medicine. For the effective and reliable clinical application of PRP in veterinary medicine, more studies are needed with regard to PRP 
characteristics as well as patient factors that influence the effectiveness of PRP. Our protocol meets the guideline of The American Red Cross and recommendations of other articles associated with the number of platelets in PRP. The PRP from our optimised protocol may produce consistently reproducible results and it will help advance comparative research and therapeutic use of PRP-related products in veterinary medicine.

\section{Additional file}

Additional file 1: WBC, neutrophil, lymphocyte and monocyte recovery after total blood centrifugation. The recovery rate of WBC, neutrophil, lymphocyte and monocyte for whole blood of each PRP1 obtained after the first centrifugation under 10 different conditions are shown in the table. (DOCX $18 \mathrm{~kb}$ )

\section{Abbreviations}

ACD: Acid citrate dextrose; EGF: Epidermal growth factor; $\mathrm{MCH}$ : Mean corpuscular haemoglobin; MCV: Mean corpuscular volume; PDGF-BB: Plateletderived growth factor-BB; PPP: Platelet-poor plasma; PRP: Platelet-rich plasma; RBC: Red blood cell; RCF: Relative centrifugal force; TGF: Transforming growth factor; VEGF: Vascular endothelial growth factor; WBC: White blood cell

\section{Acknowledgements}

Not applicable

\section{Funding}

This work was supported by Basic Science Research Program through the National Research Foundation of Korea (NRF), which is funded by the Ministry of Science, ICT \& Future Planning (NRF-2015R1C1A1A01051759) and 2015 Research Grant from Kangwon National University (No. 520150508).

\section{Availability of data and materials}

The data sets supporting the results of this article are included within the article. In addition, the raw data described in the manuscript can be requested and obtained from the corresponding author (bjkang@kangwon.ac.kr).

\section{Authors' contributions}

HSS, HMW and BJK conceived the study and performed its design and coordination. HSS performed the experiment and conducted data analysis. BJK participated in data analysis and interpretation and performed statistical analysis. HMW and BJK proofread the manuscript and gave final approval of the version to be published. All authors read and approved the final manuscript.

\section{Authors' information}

This manuscript is part of a MS thesis submitted by Dr. Shin to the College of Veterinary Medicine, Kangwon National University.

\section{Ethics approval and consent to participate}

All experimental dogs were housed at Kangwon National University (Chuncheon, Korea) in compliance with guidelines outlined by the Kangwon National University Animal Care Committee. The study protocol was approved by the Institutional Animal Care and Use Committee of Kangwon National University (Korea), Approval no.: KNU-160511-1.

\section{Consent for publication}

Not applicable

\section{Competing interests}

The authors declare that they have no competing interests.

\section{Publisher's Note}

Springer Nature remains neutral with regard to jurisdictional claims in published maps and institutional affiliations.
Received: 23 November 2016 Accepted: 19 June 2017

Published online: 26 June 2017

\section{References}

1. Eppley BL, Woodell JE, Higgins J. Platelet quantification and growth factor analysis from platelet-rich plasma: implications for wound healing. Plast Reconstr Surg. 2004;114(6):1502-8.

2. Kaplan DR, Chao FC, Stiles CD, Antoniades HN, Scher CD. Platelet alpha granules contain a growth factor for fibroblasts. Blood. 1979;53(6):1043-52.

3. Assoian RK, Komoriya A, Meyers CA, Miller DM, Sporn MB. Transforming growth factor-beta in human platelets. Identification of a major storage site, purification, and characterization. J Biol Chem. 1983;258(11):7155-60.

4. Drago L, Bortolin M, Vassena C, Taschieri S, Del Fabbro M. Antimicrobial activity of pure platelet-rich plasma against microorganisms isolated from oral cavity. BMC Microbiol. 2013;13(1):1.

5. Fortier LA, Mohammed HO, Lust G, Nixon AJ. Insulin-like growth factor-I enhances cell-based repair of articular cartilage. J Bone Joint Surg $\mathrm{Br}$. 2002;84(2):276-88.

6. Bendinelli P, Matteucci E, Dogliotti G, Corsi MM, Banfi G, Maroni P, et al. Molecular basis of anti-inflammatory action of platelet-rich plasma on human chondrocytes: mechanisms of NF-KB inhibition via HGF. J Cell Physiol. 2010:225(3):757-66.

7. Mazzocca AD, McCarthy MBR, Intravia J, Beitzel K, Apostolakos J, Cote MP, et al. An in vitro evaluation of the anti-inflammatory effects of platelet-rich plasma, ketorolac, and methylprednisolone. Arthroscopy: The Journal of Arthroscopic \& Related Surgery. 2013;29(4):675-83.

8. Everts PA, Hoffmann J, Weibrich G, Mahoney C, Schönberger J, Van Zundert A, et al. Differences in platelet growth factor release and leucocyte kinetics during autologous platelet gel formation. Transfus Med. 2006;16(5):363-8.

9. Marx RE, Carlson ER, Eichstaedt RM, Schimmele SR, Strauss JE, Georgeff KR. Platelet-rich plasma: growth factor enhancement for bone grafts. Oral Surg Oral Med Oral Pathol Oral Radiol Endod. 1998:85(6):638-46.

10. Alio $\mathrm{L}$, Abad M, Artola A, Rodriguez-Prats JL, Pastor S, Ruiz-Colecha J. Use of autologous platelet-rich plasma in the treatment of dormant corneal ulcers. Ophthalmology. 2007;114(7):1286-1293. e1.

11. Savarino L, Cenni E, Tarabusi C, Dallari D, Stagni C, Cenacchi A, et al. Evaluation of bone healing enhancement by lyophilized bone grafts supplemented with platelet gel: a standardized methodology in patients with tibial osteotomy for genu varus. J Biomed Mater Res B Appl Biomater 2006:76(2):364-72

12. Eppley BL, Pietrzak WS, Blanton M. Platelet-rich plasma: a review of biology and applications in plastic surgery. Plast Reconstr Surg. 2006;118(6):147e-59e.

13. Carter CA, Jolly DG, Worden CE, Hendren DG, Kane CJ. Platelet-rich plasma gel promotes differentiation and regeneration during equine wound healing. Exp Mol Pathol. 2003;74(3):244-55.

14. Souza TF, Andrade AL, Ferrreira G, Sakamoto SS. Albuquerque VBd, Bonfim S, Luvizotto MCR, Louzada MJQ: healing and expression of growth factors (TGF- $\beta$ and PDGF) in canine radial ostectomy gap containing platelet-rich plasma. Vet Comp Orthop Traumatol. 2012;25(6):445.

15. lacopetti I, Perazzi A, Ferrari $V$, Busetto R. Application of platelet-rich gel to enhance wound healing in the horse: a case report. Journal of Equine Veterinary Science. 2012;32(3):123-8.

16. Weibrich G, Kleis WK, Hafner G, Hitzler WE. Growth factor levels in plateletrich plasma and correlations with donor age, sex, and platelet count. J Cranio-Maxillofac Surg. 2002;30(2):97-102.

17. Weibrich G, Hansen T, Kleis W, Buch R, Hitzler W. Effect of platelet concentration in platelet-rich plasma on peri-implant bone regeneration. Bone. 2004;34(4):665-71.

18. Kawasumi $M$, Kitoh $H$, Siwicka KA, Ishiguro $N$. The effect of the platelet concentration in platelet-rich plasma gel on the regeneration of bone. J Bone Joint Surg Br. 2008;90(7):966-72

19. Kakudo N, Minakata T, Mitsui T, Kushida S, Notodihardjo FZ, Kusumoto K. Proliferation-promoting effect of platelet-rich plasma on human adiposederived stem cells and human dermal fibroblasts. Plast Reconstr Surg. 2008;122(5):1352-60.

20. Araki J, Jona M, Eto $H$, Aoi N, Kato $H$, Suga $H$, et al. Optimized preparation method of platelet-concentrated plasma and noncoagulating plateletderived factor concentrates: maximization of platelet concentration and removal of fibrinogen. Tissue Engineering Part C: Methods. 2011;18(3):176-85. 
21. Amable PR, Carias RBV, Teixeira MVT, da Cruz Pacheco l, do Amaral RJFC, Granjeiro JM, et al. Platelet-rich plasma preparation for regenerative medicine: optimization and quantification of cytokines and growth factors. Stem Cell Res Ther. 2013:4(3):1.

22. Fukaya M, Ito A. A new economic method for preparing platelet-rich plasma. Plast Reconstr Surg Glob Open. 2014;2(6):e162.

23. Perazzi A, Busetto R, Martinello T, Drigo M, Pasotto D, Cian F, Patruno M, lacopetti I. Description of a double centrifugation tube method for concentrating canine platelets. BMC Vet Res. 2013;9:146-6148-9-146.

24. Argüelles D, Carmona J, Pastor J, Iborra A, Viñals L, Martínez P, et al. Evaluation of single and double centrifugation tube methods for concentrating equine platelets. Res Vet Sci. 2006;81(2):237-45.

25. Franklin SP, Garner BC, Cook JL. Characteristics of canine platelet-rich plasma prepared with five commercially available systems. Am J Vet Res. 2015;76(9):822-7.

26. Brodniewicz-Proba T. Human plasma fractionation and the impact of new technologies on the use and quality of plasma-derived products. Blood Rev. 1991;5(4):245-57.

27. ASTM Committee F-4 on Medical and Surgical Materials and Devices, ASTM Committee F-4 on Medical and Surgical Materials and Devices. Subcommittee F04. 04 on Cardiovascular Implants and Materials: Vascular graft update: safety and performance. In Edited by Anonymous ASTM International; 1986:169-179.

28. Prothero JW: The Design of Mammals. In Edited by Anonymous Cambridge University Press; 2015;93-102.

29. Ehrenfest DMD, Rasmusson L, Albrektsson T. Classification of platelet concentrates: from pure platelet-rich plasma (P-PRP) to leucocyte-and platelet-rich fibrin (L-PRF). Trends Biotechnol. 2009;27(3):158-67.

30. Marx RE. Platelet-rich plasma: evidence to support its use. J Oral Maxillofac Surg. 2004;62(4):489-96.

31. Anitua E, Andia I, Ardanza B, Nurden P, Nurden AT. Autologous platelets as a source of proteins for healing and tissue regeneration. Thromb Haemost. 2004;91(1):4-15.

32. Everts P, Overdevest EP, Jakimowicz JJ, Oosterbos CJ, Schönberger J, Knape JT, et al. The use of autologous platelet-leukocyte gels to enhance the healing process in surgery, a review. Surg Endosc. 2007;21 (11):2063-8.

33. Monteiro SO, Lepage OM, Theoret CL. Effects of platelet-rich plasma on the repair of wounds on the distal aspect of the forelimb in horses. Am J Vet Res. 2009;70(2):277-82.

34. Maia L, de Souza MV, Júnior JIR, de Oliveira AC, Alves GES, dos Anjos Benjamin $L$, et al. Platelet-rich plasma in the treatment of induced tendinopathy in horses: histologic evaluation. Journal of equine veterinary science. 2009;29(8):618-26.

35. Frye CW, Enders A, Brooks MB, Struble AM, Wakshlag JJ. Assessment of canine autologous platelet-rich plasma produced with a commercial centrifugation and platelet recovery kit. Vet Comp Orthop Traumatol. 2016;29(1):14-9.

36. Kurita M, Aiba-Kojima E, Shigeura T, Matsumoto D, Suga H, Inoue K, et al. Differential effects of three preparations of human serum on expansion of various types of human cells. Plast Reconstr Surg. 2008;122(2):438-48.

37. Schnabel LV, Mohammed HO, Miller BJ, McDermott WG, Jacobson MS, Santangelo KS, et al. Platelet rich plasma (PRP) enhances anabolic gene expression patterns in flexor digitorum superficialis tendons. J Orthop Res. 2007;25(2):230-40.

38. McCarrel T, Fortier L. Temporal growth factor release from platelet-rich plasma, trehalose lyophilized platelets, and bone marrow aspirate and their effect on tendon and ligament gene expression. J Orthop Res. 2009;27(8):1033-42.

39. McCarrel TM, Minas T, Fortier LA: Optimization of leukocyte concentration in platelet-rich plasma for the treatment of tendinopathy. J Bone Joint Surg Am. 2012;94(19):e143(1-8)

40. Misso S, Feola B, Marotta C, Graziano D, Fratellanza A, Minerva V, et al. Sottopopolazioni leucocitarie in concentrati piastrinici da aferesi e da plasma ricco di piastrine. La trasfusione del sangue. 2001;46(4):258-62.

41. Zimmermann R, Reske S, Metzler P, Schlegel A, Ringwald J, Eckstein R. Preparation of highly concentrated and white cell-poor platelet-rich plasma by plateletpheresis. Vox Sang. 2008;95(1):20-5.

42. Castillo TN, Pouliot MA, Kim HJ, Dragoo JL. Comparison of growth factor and platelet concentration from commercial platelet-rich plasma separation systems. Am J Sports Med. 2011;39(2):266-71.

43. Textor JA, Tablin F. Activation of equine platelet-rich plasma: comparison of methods and characterization of equine autologous thrombin. Vet Surg. 2012:41(7):784-94.

\section{Submit your next manuscript to BioMed Central and we will help you at every step:}

- We accept pre-submission inquiries

- Our selector tool helps you to find the most relevant journal

- We provide round the clock customer support

- Convenient online submission

- Thorough peer review

- Inclusion in PubMed and all major indexing services

- Maximum visibility for your research

Submit your manuscript at www.biomedcentral.com/submit 